\title{
A community-based Falls Management Exercise Programme (FaME) improves balance, walking speed and reduced fear of falling
}

\author{
Pui Yee Yeung ${ }^{1}$, Wayne Chan $^{2}$ and Jean Woo ${ }^{1}$ \\ ${ }^{1}$ Department of Medicine and Therapeutics, The Chinese University of Hong Kong, Shatin, Hong Kong \\ ${ }^{2}$ Hong Kong Society for the Aged, Hong Kong
}

\begin{abstract}
Background: Although effective community falls prevention programmes for the older persons have been described, challenges remain in translating proven interventions into daily practice. Aim: To evaluate the efficacy, feasibility and acceptability of a falls prevention programme that can be integrated into daily activities in a group of community-dwelling older adults with risk of falling. Method: A cohort study with intervention and comparison groups was designed to evaluate a 36-week group-based falls prevention exercise programme (FaME) in the community setting. Participants were aged 60 years or older, had fallen in the past 12 months, had fear of falling with avoidance of activities or had deficits in balance control. Primary outcome measures included assessment of balance control and mobility; secondary outcome measures included level of physical activity, assessment of fear of falling and health-related quality of life. Results: There were 48 and 51 participants in the intervention and comparison groups, respectively. There were improvements in measurements of balance, walking speed and self-efficacy. The drop out rate was low $(14.6 \%$ and $3.9 \%$ from the intervention and comparison groups, respectively). Overall compliance in the intervention group was $79 \%$. Factors that motivated continued participation include the regular and long-term nature of the programme helping to reinforce their exercise habits, the simplicity of movements and friendliness of the group. Conclusion: The FaME programme improves balance, walking speed and reduces fear of falling. It could be widely promoted and integrated into regular health and social activities in community settings.
\end{abstract}

Key words: balance; community; falls prevention; self-efficacy; walking speed

Received 19 September 2012; revised 11 November 2013; accepted 29 December 2013; first published online 30 January 2014

\section{Introduction}

Falls is one of the most common syndromes among older people, affecting about one-third of community-dwelling older people aged 65 and above (Gillespie et al., 2009). Effective community falls prevention programmes for the older persons with good uptake are important for reducing health-care system demands with population

Correspondence to: Professor Jean Woo, Department of Medicine \& Therapeutics, Prince of Wales Hospital, Shatin, N.T. Hong Kong, China. Email: jeanwoowong@cuhk.edu.hk ageing (WHO, 2008). Although multi-component group exercises targeting on strength, balance, flexibility and endurance have been shown to be equally or even more effective compared with more complicated multi-factorial targeted interventions among community-dwelling older people with risk of falling (Baker et al., 2007; Sherrington et al., 2008; Gillespie et al., 2009; Petridou et al., 2009), challenges remain in translating proven interventions into daily practice.

The first challenge is diffusing knowledge of the best available evidence (Ganz et al., 2008). ProFaNE (Prevention of Falls Network Europe), 
a collaborative project that links researchers, clinicians and members of the public across Europe and worldwide to promote the best practice through evidence development, represents such a model (Skelton et al., 2004; Skelton and Todd, 2007). The second challenge concerns the extent to which the proven interventions reach their target population. For instance, a survey regarding falls service in the United Kingdom revealed that only $13 \%$ of falls prevention programmes in health and social care settings included exercise classes (Martin, 2009). It is shown that the proven effective intervention, exercise programme, did not have an optimal coverage in the falls service. Subsequent challenges concern the feasibility of implementing proven interventions in appropriate settings, acceptability of the interventions by target participants and sustainability of the interventions (Robitaille and Gauvin, 2008). Recent studies suggest that effective falls prevention programmes should be sustainable, enjoyable and effective over the long term, and integration into daily activities at home is important (Clemson et al., 2012; Morris, 2012).

A previous study on the older Hong Kong population showed that while exercise programmes may target lower limb weakness and unsteady gait, identified as the two major predisposing factors for falls (Yeung et al., 2009), uptake rate was low and the barriers to participation were mainly because of low perceived needs for falls prevention and concerns about transportation among participants (Wong et al., 2011). To overcome these barriers, exercise programmes for falls prevention could be incorporated as part of the daily activity programmes of community or social care centres; adding diverse training components to enhance health, enjoyment, mood and independence rather than just emphasizing reducing risk of falling (Yardley et al., 2006). Furthermore, peer support and social network in such settings may improve uptake and maintenance of such exercises (McInnes and Askie, 2004). Current existing community-based exercise programmes for older people are short term, held on an on-off basis and may not be evidence based in programme design. We designed a falls prevention programmes that can be integrated into daily activities, is enjoyable and long lasting based on the multi-factorial exercise protocol for falls prevention (FaME) proposed by Skelton and
Dinan (Skelton and Dinan, 1999), and evaluated the efficacy, feasibility and acceptability in a group of community-dwelling older adults with risk of falling.

\section{Method}

A cohort study with intervention and comparison groups was designed to evaluate the 36-week group-based falls prevention exercise programme in the community setting. For participants, inclusion criteria were: aged 60 years or older; living in the community; able to walk without aids/with stick or quadripod; met any one of the following criteria - had at least one fall in last 12 months, had fear of falling with avoidance of activities or had deficits in balance control (time-up-and-go test $>12 \mathrm{~s}$, or Berg Balance Scale-Short Form score $\leqslant 20$ ). Exclusion criteria were: (i) limitation in mobility requiring manual assistance in walking and transfer (eg, as a result of previous stroke and fracture); (ii) hearing and vision impairments affecting communication; and (iii) cognitive impairment (Chinese Mini Mental State Examination score <19).

Potential subjects for the intervention group were recruited from advertisements placed in public places in the community, from a church group, from hospital wards and outpatients and from public carnivals for the elderly. Subjects for the comparison group were recruited from two elderly centres. Members of the two centres who underwent the screening tests and met the criteria were invited to join the study as comparison group participants.

\section{Intervention and comparison group programmes}

Forty-eight participants enrolled in the intervention group and attended a 36-week FAME, based on the multi-factorial exercise protocol for falls prevention by Skelton and Dinan (Skelton and Dinan, 1999). The programme was held in an integrated primary health and social care centre located in a public housing estate. Seven participants dropped out before completing the programme (Figure 1). The programme was run in groups of six participants for one hour per session, one session per week, and led by one to two exercises instructors. The sessions consisted of warm-up, muscle strengthening, functional mobility training,

Primary Health Care Research \& Development 2015; 16: 138-146 


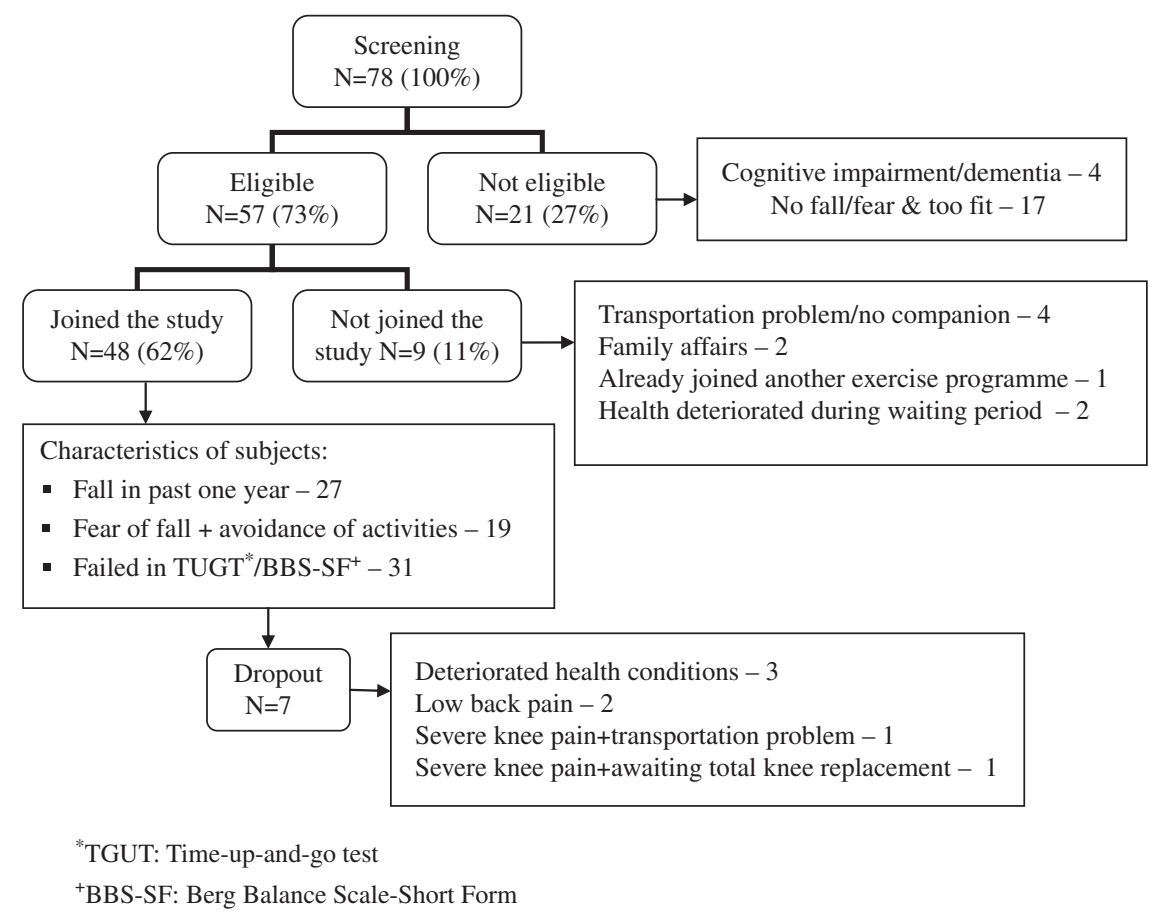

Figure 1 Flow of intervention group.

gait training, balance control and cool-down exercises. The 36-week exercise programme was divided into three phases:

1. Skilling up (week 1-12) - focused on adaptation of exercise technique and safety issues, adaptation of muscular function, practicing get-up-from-floor technique, improving upper and lower limb strength, and gait training.

2. Training gain I (week 13-24) - progressive training in muscle strength, functional floor work, dynamic balance in free standing position, eye-hand and foot coordination and walking function. Involved multi-task performance during balance and walking training.

3. Training gain II and maintenance (week 25-36) further improved muscle strength and consolidated the progress, balance and walking function. Challenged visual, somatosensory and vestibular mechanism of balance during balance and walking training. Reviewed the exercises learned in the programme.

In addition, simple home exercises were taught at the beginning of each phase and revised halfway through each phase. Home exercise log books were distributed to participants to record their practice of home exercise.

Furthermore, participants were asked to set goals related to their exercise practice, physical functioning and performance in falls prevention at the beginning of the second phase. The goals were set based on the discussion and mutual agreement between participants and exercise instructors, according to the present physical condition and the desired achievement. The goals might be revised at the end of the second phase, and the problems that hinder participants to achieve their own goals were solved with the guidance of exercise instructors. The goals and the level of achievement were recorded and assessed using Goal Attainment Scaling (GAS), which was developed by Kiresuk et al. (1994).

For the comparison group, 51 participants were enrolled and they continued their usual activities in the community for 36 weeks. Two of them dropped out before the end of the study period (Figure 2). They were allowed to join any activity in the elderly centres within the study period. 


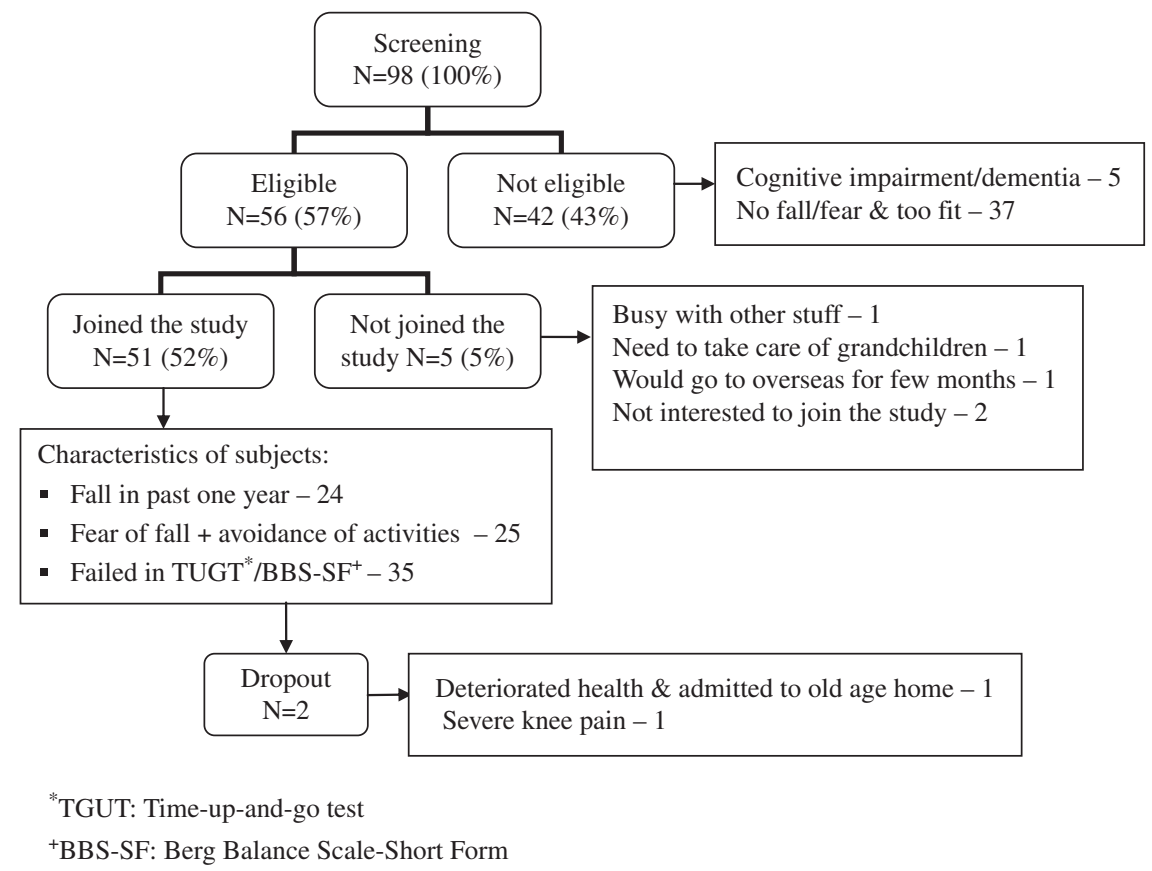

Figure 2 Flow of comparison group.

At the end of the study period, they were invited to join an eight-session fall prevention exercise programme if they wished.

\section{Evaluation}

Participants in both intervention and comparison groups were assessed at baseline, 12, 24 and 36 weeks. Screening tests included Chinese Mini Mental State Examination (CMMSE; Chiu et al., 1994), fear of falling (one question being asked: Are you afraid of falling?), avoidance of activities (one question being asked: Do you avoid certain activities because you are afraid of falling?), Berg Balance Scale-Short Form (BBS-SF; Chou et al., 2006), Time-up-and-go test (TUGT; Podsiadlo and Richardson, 1991) and falls history in the last one year. At baseline, information was collected regarding age, gender, past medical history, medications, fall history and exercise habit. Primary outcome measures included assessment of balance control and mobility by TUGT, Berg Balance Scale (BBS; Berg et al., 1989) and six-min walking test (6MWT; Guyatt et al., 1985). Secondary outcome measures included Physical Activity Scale for the Elderly (PASE) for measuring level of physical activity (Washburn et al., 1993); Fall Efficacy Scale-International (FES-I) for assessing fear of falling (Yardley et al., 2005); and health-related quality of life assessed using the SF-12 (physical component summary score PCS and mental component summary score MCS; Ware et al., 1996). Although not included as an outcome measure, falls were recorded prospectively in terms of total number, number requiring medical attention, number of fallers and number of recurrent fallers. For the intervention group, falls were recorded by directly asking participants during each exercise session. For the comparison group, fall calendars were distributed to individual participants to record their falls and checked by telephone every two weeks.

Assessments were carried out at baseline, 12, 24 and 36 weeks, by a team of research staff trained on a standardized approach for completing the questionnaires and conducting the various tests. The exercise instructors were excluded from the assessment team when conducting assessments for the intervention groups led by them to minimize bias. Intervention group participants were also invited to give their feedback, comments and suggestions to the programme staff. 


\section{Sample size}

On the basis of findings from a pilot study, where the effect sizes of various outcomes ranged from 0.70 to $1.47,33$ participants were needed in each intervention and comparison groups to detect significant difference based on an $\alpha$ of $5 \%$ (twosided) and a power of $80 \%$. Taking a dropout rate of $20 \%$ (which occurred in the pilot study) into account, a minimum of 42 participants was required for each group.

\section{Statistical analysis}

Demographics and scores of outcome measures at baseline were compared using the $\chi^{2}$-test for categorical variables or unpaired Student's $t$-test for continuous variables. Repeated measure of analysis of variance (RANOVA) was used to compare the change of outcome measures between the intervention and comparison groups over time. Repeated measure of analysis of covariance (RANCOVA) was used to compare the change of outcome measures between the intervention and comparison groups over time controlled for covariates at baseline. MannWhitney $U$ test was used to compare the difference between the intervention and comparison groups in the number of falls and number of falls requiring medical attention within the study period. $\chi^{2}$-test was used to examine significant difference between the intervention and comparison groups in the number of fallers and recurrent fallers within the study period. Significant difference was accepted at $P<0.05$ for all analyses. All analyses were performed using SPSS version 16.0 for Windows (SPSS Inc. Chicago, IL, USA).

\section{Results}

Seven of the 48 participants $(14.6 \%)$ from the intervention group and two of the 51 participants $(3.9 \%)$ from the comparison group dropped out of the study. The overall attendance rate for those intervention group participants who completed the exercise programme was $79.0 \%$. Baseline characteristics were similar between the intervention and comparison groups (Table 1), with the exception of a higher percentage of participants performing stretching and mobilizing exercise $(P<0.001)$, a higher amount of exercise $(P=0.005)$ and a lower CMMSE score $(P=0.007)$ in the comparison compared with the intervention group. These three variables were included as covariates when comparing the change of outcome measures between groups over time.

Table 2 shows the primary (TUGT, BBS and 6MWT) and secondary (PASE, FES-I, SF-12 PCS and SF-12 MCS) outcome measures for both groups at different time points. Repeated measures ANOVA revealed a significant time effect and a significant group effect (by using the interaction term) for all the primary outcome measures from baseline to 36 weeks (Table 2). The intervention group had significantly better performances in TUGT, BBS and 6MWT than the comparison group over 36 weeks. Pairwise comparisons indicated that the results of TUGT, BBS and 6MWT at baseline were significantly different from those results at 12, 24 and 36 weeks for the intervention group. After controlling for covariates (age, sex, participation in stretching and mobilizing exercise, exercise time per week and CMMSE score), significant differences were found for BBS $(P=0.001)$ and 6MWT $(P=0.001)$ but not TUGT between the groups over time.

Repeated measures ANOVA revealed a significant time effect and a significant group effect (by using the interaction term) for PASE, FES-I and SF-12 MCS from baseline to 36 weeks (Table 2). For PASE, the intervention group had significantly higher levels of physical activity than the comparison group over 36 weeks, and pairwise comparisons indicated that the result of PASE at baseline was significantly different from those results at 12 and 24 weeks for the intervention group. For FES-I, the intervention group had less fear of falling than the comparison group over 36 weeks and pairwise comparisons indicated that the result of FES-I at baseline was significantly different from those results at 24 and 36 weeks for the intervention group. Among the secondary outcome measures, significant difference was only found in FES-I $(P=0.013)$ between the groups over time after controlling for covariates (age, sex, participation in stretching and mobilizing exercise, exercise time per week and CMMSE score).

There was no significant difference between the groups in the number of falls (18/41 versus 20/49), the number of falls requiring medical attention during the study period (3/41 versus 6/49), the percentage of fallers $(27 \%$ versus $31 \%)$ and the 
Table 1 Demographics and outcome measures at baseline

\begin{tabular}{|c|c|c|c|}
\hline Demographics & $\begin{array}{l}\text { Intervention } \\
\text { group }(n=48)\end{array}$ & $\begin{array}{l}\text { Comparison } \\
\text { group }(n=51)\end{array}$ & $P$-value \\
\hline Age & $73.94(8.00)$ & $76.80(8.02)$ & 0.078 \\
\hline$\%$ Female & $72.9 \%$ & $88.2 \%$ & 0.053 \\
\hline Total number of diseases & $2.25(1.38)$ & $2.31(1.21)$ & 0.807 \\
\hline Total number of medications & $1.92(2.06)$ & $2.45(2.22)$ & 0.218 \\
\hline$\%$ Having regular exercise & $72.9 \%$ & $90.2 \%$ & 0.083 \\
\hline$\%$ Doing stretching and mobilizing exercise & $54.2 \%$ & $90.2 \%$ & $<0.001$ \\
\hline$\%$ Doing strengthening exercise & $12.5 \%$ & $5.9 \%$ & 0.252 \\
\hline \% Doing aerobic exercise & $64.6 \%$ & $70.6 \%$ & 0.523 \\
\hline$\%$ Doing balance exercise & $25.0 \%$ & $39.2 \%$ & 0.131 \\
\hline Exercise time per week (min) & $280.57(276.47)$ & 468.49 (364.69) & 0.005 \\
\hline$\%$ With previous fracture & $12.5 \%$ & $13.7 \%$ & 0.857 \\
\hline$\%$ Faller in the last year & $52.1 \%$ & $49.0 \%$ & 0.761 \\
\hline Number of falls in the last year & $0.90(1.63)$ & $0.59(0.67)$ & 0.217 \\
\hline$\%$ Fall-related medical attention in the last year & $27.1 \%$ & $27.5 \%$ & 0.967 \\
\hline$\%$ Fall-related A\&E visit in the last year & $25.0 \%$ & $11.8 \%$ & 0.088 \\
\hline$\%$ Fall-related admission in the last year & $6.3 \%$ & $3.9 \%$ & 0.597 \\
\hline LOS for fall-related admission in the last year (days) & $0.23(0.93)$ & $0.69(4.25)$ & 0.467 \\
\hline$\%$ Fall-related soft-tissue injury in the last year & $37.5 \%$ & $37.3 \%$ & 0.980 \\
\hline$\%$ Fall-related fracture in the last year & $2.1 \%$ & $7.8 \%$ & 0.191 \\
\hline CMMSE & $27.67(2.45)$ & $26.16(2.96)$ & 0.007 \\
\hline TUGT (s) & $12.15(2.95)$ & $13.65(5.71)$ & 0.101 \\
\hline BBS & $50.67(4.09)$ & $50.45(4.58)$ & 0.806 \\
\hline 6MWT (m) & $391.21(74.65)$ & $365.28(101.48)$ & 0.154 \\
\hline PASE & $82.72(28.35)$ & $79.34(36.57)$ & 0.610 \\
\hline FES-I & $29.56(8.82)$ & $29.10(11.11)$ & 0.819 \\
\hline SF-12 PCS & $41.50(9.42)$ & $41.23(10.99)$ & 0.895 \\
\hline SF-12 MCS & $50.30(10.17)$ & $50.74(12.15)$ & 0.845 \\
\hline
\end{tabular}

CMMSE = Chinese Mini Mental State Examination; TUGT = Time-up-and-go test; BBS = Berg Balance Scale; 6MWT = six-min walking test; PASE = Physical Activity Scale for the Elderly; FES-I = Fall Efficacy Scale-International; PCS = physical component summary score; MCS = mental component summary score.

number of recurrent fallers (17\% versus 20\%) between the intervention and comparison groups. However, the numbers were too small and the study was not powered to address falls as a primary outcome measure.

Feedback was collected from the intervention group participants regarding their views towards the key elements that motivated their participation. These include increased tolerance for doing housework and improved walking stability, the regular and long-term nature of the programme, which helped to reinforce their exercise habits and integrate the home exercise in their daily routine, the manageable level of difficulty for the programme allowing them to master the different levels of exercise progressively, the simplicity of movements (compared with Tai Chi), and the comfortable and friendly environment with a group of peers, which enhanced their motivation.

\section{Discussion}

This programme achieved improvement in balance, exercise tolerance and falls self-efficacy, had a low dropout rate, high attendance rate and favourable participant feedback. The study suggests that it may be a suitable programme for community-dwelling older adults with risk of falling to be integrated into local community activities. The high adherence rate of $85 \%$ over a period of 36 weeks is comparable to the average adherence rate $(\sim 70 \%)$ at 12 months for walking and class-based exercise interventions targeted at falls prevention found in a review (Nyman and Victor, 2012). The 'group' nature of the programme fostering a sense of belonging and promoting peer support among the participants may contribute to the high adherence rate (Skelton, 2001). 


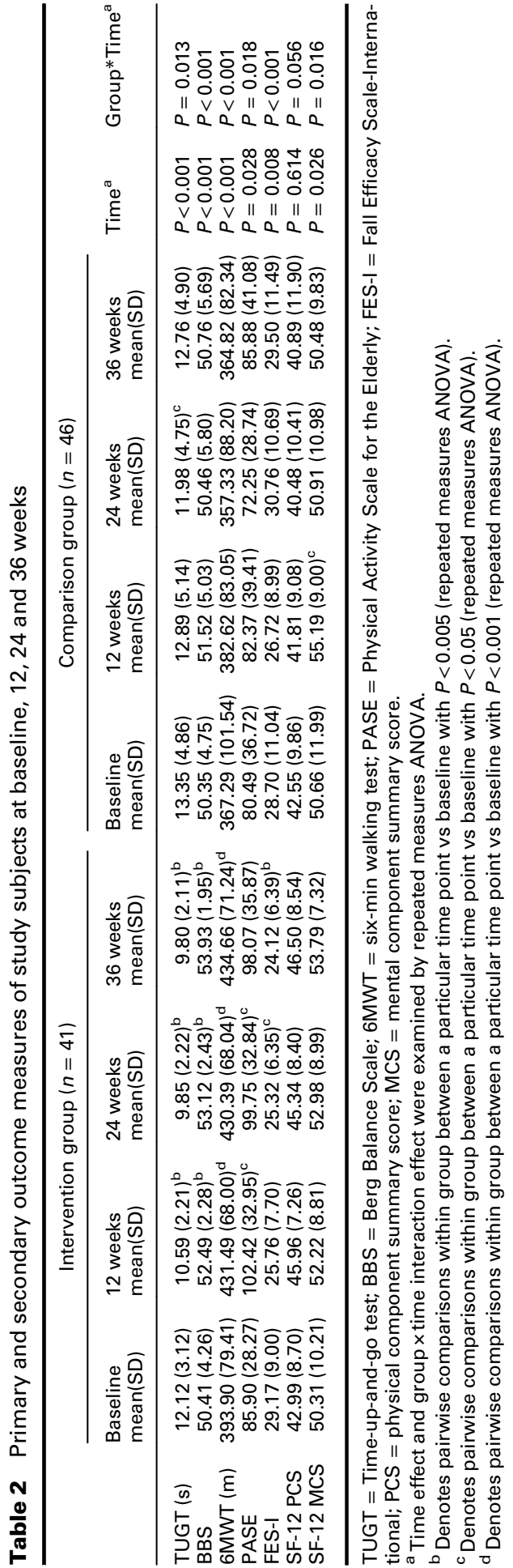

Although the intervention group had improved primary outcomes, no difference in the number of falls was observed. Various factors may explain this finding: the study was not powered to detect a difference in falls rate between the groups; some of the subjects were not fallers before joining this study they were only afraid of falling; the exercise programme did not address some of the known risk factors for falls such as medical or environmentalrelated risk factors, or engagement in risky behaviours; accuracy of falls ascertainment may differ between the groups as the control group subjects were contacted via phone once every two weeks to check whether any fall has occurred and those telephone contacts may serve as an intervention itself to increase their awareness in falls prevention.

The challenge ahead concerns a wider implementation of the programme in the local community settings such as elderly centres, primary care centres and community centres in various local districts. Understanding potential barriers to adoption and proposing ways to minimize those barriers are important steps before successful implementation. These may include the requirement that only specified disciplines can lead these exercise groups. Our study showed that it is possible for the staff to be trained by a health-care professional to follow protocol: two of the exercise instructors in the present study received coaching from an allied health professional. Another barrier is a common misconception that effective programmes require expensive equipment, whereas our programme used only lowcost items such as therabands (elastic resistance bands), steps, fit-balls and mats. There are also concerns that participants may have injuries while performing the exercises. Use of hip protectors during exercise may be a useful way to reduce the risk of fractures and improve confidence for both participants and instructors (Skelton and Dinan, 1999; Skelton and Todd, 2004).

There are some limitations in the present study. Recruitment for the intervention and comparison groups were from different community sources. We avoided recruiting participants for intervention and comparison groups from the same centres, because of the possibility of 'contamination'. For the comparison group recruited from two centres, the activity programmes for these centres vary, and there was little physical activity for one of these centres. The other centre encouraged members to do stretching and mobilizing exercises. 
For participants of the FaME programme, 20 of these also participated in social centre and church group health-oriented activities. However, we did not record this information in detail. Factors other than existing exercise programmes may also contribute to the baseline differences and outcomes between the intervention and comparison group. It is possible that there could be less room for improvement in the comparison group if their baseline functioning is higher. As for randomized controlled trials, baseline differences may still occur between the two groups, and adjustment for baseline values that are different would be an accepted analytical method, and we have attempted to do the same in the statistical analysis. There may be recall bias in retrospective reporting of falls. Despite these limitations, the study identified certain features of exercise programmes that may be prerequisites for incorporation into regular community activities that have both health and social benefits, which should not be overlooked as they may help promote participation and sustain long-term adherence (Skelton, 2001; Schneider et al., 2003). The findings also suggest that the FaME programme appears to be feasible and acceptable for the community-dwelling older adults with the risk of falling, and could be widely promoted and integrated into regular health and social activities in community settings.

\section{Ethical considerations, funding support and conflict of interest declaration}

The study was approved by the local ethics committee and all the participants in both intervention and control groups provided a written informed consent before participation. They were informed of the purpose of the study, anonymity and confidentiality of the data, and of the right to withdraw from the study at any time. The study received funding support from the SH Ho Centre for Gerontology and Geriatrics. None of the authors had any conflict of interest.

\section{Acknowledgement}

The authors thank the staff of the Tai Po Cadenza Hub for providing the venue for this study.

\section{References}

Baker, M.K., Atlantis, E. and Fiatarone Singh, M.A. 2007: Multi-modal exercise programs for older adults. Age and Ageing 36, 375-81.
Berg, K., Wood-Dauphinee, S., Williams, J.I. and Gayton, D. 1989: Measuring balance in the elderly: preliminary development of an instrument. Physiotherapy Canada 41, 304-11.

Chiu, H.F., Lee, H.B., Chung, W.S. and Kwong, P.K. 1994: Reliability and validity of the Cantonese version of minimental state examination: a preliminary study. Journal of Hong Kong College of Psychiatry 4, S25-28.

Chou, C.Y., Chien, C.W., Hsueh, I.P., Sheu, C.F., Wang, C.H. and Hsieh, C.L. 2006: Developing a short form of the Berg Balance Scale for people with stroke. Physical Therapy 86, 195-204.

Clemson, L., Fiatarone Singh, M.A., Bundy, A., Cumming, R.G., Manollaras, K., O'Loughlin, P. and Black, D. 2012: Integration of balance and strength training into daily life activity to reduce rate of falls in older people (the LiFE study): randomised parallel trial. British Medical Journal 345, e4547.

Ganz, D.A., Alkema, G.E. and Wu, S. 2008: It takes a village to prevent falls: reconceptualizing fall prevention and management for older adults. Injury Prevention 14, 266-71.

Gillespie, L.D., Robertson, M.C., Gillespie, W.J., Lamb, S.E., Gates, S., Cumming, R.G. and Rowe, B.H. 2009: Interventions for preventing falls in older people living in the community. Cochrane Database Systematic Reviews 2, CD007146.

Guyatt, G.H., Sullivan, M.J., Thompson, P.J., Fallen, E.L., Pugsley, S.O., Taylor, D.W. and Berman, L.B. 1985: The 6-minute walk: a new measure of exercise capacity in patients with chronic heart failure. Canadian Medical Association Journal 132, 919-23.

Kiresuk, T.J., Smith, A. and Cardillo, J.E. 1994. Goal attainment scaling: Applications, theory, and measurement. Hillsdale, N.J.: L. Erlbaum Associates.

Martin, F.C. 2009: Next steps for falls and fracture reduction. Age and Ageing 38, 640-43.

McInnes, E. and Askie, L. 2004: Evidence review on older people's views and experiences of falls prevention strategies. Worldviews on Evidience-Based Nursing 1, 20-37.

Morris, M.E. 2012: Preventing falls in older people. British Medical Journal 345, e4919.

Nyman, S.R. and Victor, C.R. 2012: Older people's participation in and engagement with falls prevention interventions in community settings: an augment to the Cochrane systematic review. Age and Ageing 41, 16-23.

Petridou, E.T., Manti, E.G., Ntinapogias, A.G., Negri, E. and Szczerbinska, K. 2009: What works better for communitydwelling older people at risk to fall?: a meta-analysis of multifactorial versus physical exercise-alone interventions. Journal of Aging and Health 21, 713-29.

Podsiadlo, D. and Richardson, S. 1991: The timed "Up \& Go": a test of basic functional mobility for frail elderly persons. Journal of the American Geriatrics Society 39, 142-48.

Robitaille, Y. and Gauvin, L. 2008: Fall prevention in older adults: towards an integrated population-based perspective. Injury Prevention 14, 147-48.

Schneider, J.K., Eveker, A., Bronder, D.R., Meiner, S.E. and Binder, E.F. 2003: Exercise training program for older adults. Incentives and disincentives for participation. Journal of Gerontological Nursing 29, 21-31. 
Sherrington, C., Whitney, J.C., Lord, S.R., Herbert, R.D., Cumming, R.G. and Close, J.C. 2008: Effective exercise for the prevention of falls: a systematic review and meta-analysis. Journal of the American Geriatrics Society 56, 2234-243.

Skelton, D.A. 2001: Effects of physical activity on postural stability. Age and Ageing 30, 33-39.

Skelton, D.A., Becker, C., Lamb, S.E., Close, J.C., Zijlstra, W., Yardley, L. and Todd, C. 2004: Prevention of Falls Network Europe: a thematic network aimed at introducing good practice in effective falls prevention across Europe. European Journal of Ageing 1, 89-94.

Skelton, D.A. and Dinan, S.M. 1999: Exercise for falls management: rationale for an exercise programme aimed at reducing postural instability. Physiotherapy Theory and Practice 15, 105-20.

Skelton, D.A. and Todd, C.J. 2004. What are the main risk factors for falls amongst older people and what are the most effective interventions to prevent these fallsCopenhagenHealth Evidence Network: World Health Organization. Retrieved 24 August 2012 from http://www.euro.who.int/_ data/assets/pdf_file/0018/74700/E82552.pdf

Skelton, D.A. and Todd, C.J. 2007: Prevention of Falls Network Europe: a thematic network aimed at introducing good practice in effective falls prevention across Europe. Four years on. Journal of Musculoskeletal \& Neuronal Interactions 7, 273-78.

Ware, J. Jr., Kosinski, M. and Keller, S.D. 1996: A 12-Item Short-Form Health Survey: construction of scales and preliminary tests of reliability and validity. Medical Care 34, 220-33.

Washburn, R.A., Smith, K.W., Jette, A.M. and Janney, C.A. 1993: The Physical Activity Scale for the Elderly (PASE): development and evaluation. Journal of Clinical Epidemiology 46, 153-62.

WHO. 2008. WHO global report on falls prevention in older age. Geneva: World Health Organization. Retrieved 24 August 2012 from http://www.who.int/ageing/publications/Falls_ prevention7March.pdf

Wong, E.L., Woo, J., Cheung, A.W. and Yeung, P.Y. 2011: Determinants of participation in a fall assessment and prevention programme among elderly fallers in Hong Kong: prospective cohort study. [Research Support, Non-U.S. Gov't]. Journal of Advanced Nursing 67, 763-73.

Yardley, L., Beyer, N., Hauer, K., Kempen, G., Piot-Ziegler, C. and Todd, C. 2005: Development and initial validation of the Falls Efficacy Scale-International (FES-I). Age and Ageing 34, 614-19.

Yardley, L., Bishop, F.L., Beyer, N., Hauer, K., Kempen, G.I., Piot-Ziegler, C., Todd, C.J., Cuttelod, T., Horne, M., Lanta, K. and Holt, A.R. 2006: Older people's views of fallsprevention interventions in six European countries. The Gerontologist 46, 650-60.

Yeung, P.Y., Woo, J., Yim, V.W. and Rainer, T.H. 2009: Heterogeneity of health profiles of older people presenting to an accident and emergency department with a fall. International Journal of Gerontology 3, 156-62. 